\title{
DYNAMIC BIT ALLOCATION AND DIFFERENTIAL QUANTIZATION IN FEEDBACK LINK FOR ROBUST POWER ALLOCATION IN MULTIUSER OPPORTUNISTIC BEAMFORMING
}

\author{
Nizar Zorba ${ }^{1}$, Antonio Pascual-Iserte ${ }^{1,2}$, Ana I. Pérez Neira ${ }^{1,2}$ \\ ${ }^{1}$ Centre Tecnològic de Telecomunicacions de Catalunya (CTTC) - Spain \\ ${ }^{2}$ Dept. of Signal Theory and Communications - Universitat Politècnica de Catalunya (UPC) - Spain \\ email: nizar.zorba@cttc.es, antonio.pascual@upc.edu, anuska@gps.tsc.upc.edu
}

\begin{abstract}
Multi-antenna systems provide advantages in terms of link quality and multiuser access capabilities, which can be achieved by using schemes as multibeam opportunistic beamforming. This scheme only requires partial channel state information at the transmitter (CSIT) in terms of the equivalent channel moduli. This paper deals with the problem of feeding back the CSI from the multiple receivers to the base station (BS) transmitter through limited capacity feedback links. This involves a quantization and a delay that produces errors in the CSIT. In this sense, the proposed scheme in this paper is based on a robust design that takes into account these errors. Additionally, a dynamic bit allocation in the feedback among the users is derived, and combined with differential quantization to minimize the transmit power. It considers both the users' sensitivities to the quantization errors and the Doppler frequencies due to the mobility. Simulation results show the benefits from using such schemes.
\end{abstract}

\section{INTRODUCTION}

In an effort to benefit from both the opportunistic scheduling gain and the multiple-input-multiple-output (MIMO) technology in multiuser scenarios, the multibeam opportunistic beamforming (MOB) strategy has been suggested [1] to boost the wireless link capabilities, showing a high performance and a low complexity design. A characterizing feature of this scheme is that it operates with partial CSIT in the sense that it is not necessary to have all the multi-antenna channel knowledge, but only the scalar channel moduli information.

MOB can be operated and adopted to fulfill the quality of service $(\mathrm{QoS})$ requirements demanded by the users for their correct operation in terms, for example, of a minimum signal to noise and interference ratio (SNIR) per user. In this sense, an attractive transmission scheme is accomplished by meeting such requirements while minimizing the total transmit power [2].

Achieving a QoS compliant system in $\mathrm{MOB}$ requires taking into account the cross interference terms that this scheme originates in the multiuser scenario, as each user receives an interference component from each one of the generated beams [1]. This paper presents the QoS fulfillment of MOB as an optimization problem that guarantees the QoS for each serviced user, while the total transmit power is minimized. Another handicap for QoS relates to the CSIT quality, that in practical scenarios is not perfect due to fast fading, limited duration of the training step, and/or feedback quantization. Motivated by this

This work was partially supported by the Catalan Government under grant 2005SGR-00996; by the Spanish Government under projects TEC2005-08122-C03 (ULTRA-PROCESS) and 2A103 (MIMOWA) from MEDEA+ program (PROFIT FIT-330225-2007-2), and by the European Commission under project NEWCOM++ (216715). fact, the paper presents a practical power allocation scheme that is robust to the uncertainty in the equivalent scalar channel moduli information. Therefore, the QoS satisfaction with minimum transmit power is formulated in imperfect CSIT scenarios, where this optimization problem is solved in a closed form expression.

The CSIT uncertainty comes from two main sources, the first one relates to the quantization performed over the feedback channel, as only a finite number of feedback bits can be used [3]. The second cause of uncertainty is due to the terminals mobility, as the channel may have changed within the feedback process [4]. The two uncertainty sources are considered in this work, giving a deep comprehension of their effects on the system performance. An interesting observation is that the number of feedback bits has a direct impact on the uncertainty level, where the feedback bits constitute a system resource that needs for optimization. In this paper, a dynamic bit allocation process over the feedback links is suggested combined with a differential quantization strategy [5], where this scheme is based on the sensitivity of each user towards the feedback uncertainty.

The remainder of this paper is organized as follows: while section 2 deals with the signal and channel models, in section 3 a review of the MOB procedure is presented, where a robust power allocation for QoS fulfillment with imperfect CSIT is discussed. Section 4 presents the proposed dynamic feedback bits allocation policy, followed by the simulations and conclusions in section 5 and 6 .

\section{SIGNAL AND CHANNEL MODELS}

We focus on the downlink channel where $N$ single-antenna receivers are being served by a transmitter at the BS provided with $n_{t}$ transmit antennas, and supposing that $N \geq n_{t}$. The flat fading multiantenna channel between the BS and the $i$ th receiver during the $t$ th time slot is represented by the row complex vector $\mathbf{h}_{i}(t) \in \mathbb{C}^{1 \times n_{t}}$ under a quasi static block fading model. The channel shows a temporal correlation between the consecutive slots (indexed by $t$ ), which is modeled by

$$
\mathbf{h}_{i}(t)=\rho_{i} \mathbf{h}_{i}(t-1)+\sqrt{1-\rho_{i}^{2}} \mathbf{w}_{i}(t),
$$

where both $\mathbf{h}_{i}(t-1)$ and $\mathbf{w}_{i}(t)$ are i.i.d. random vectors composed of $n_{t}$ independent complex Gaussian entries $\sim \mathcal{C N}\left(0, \sigma_{h_{i}}^{2}\right)$, with $\rho_{i}$ defining the level of temporal correlation and $\sigma_{h_{i}}^{2}$ being the channel variance. The correlation $\rho_{i}$ is directly related to the Doppler frequency of the user $\left(f_{D i}\right)$ and the time difference between consecutive slots $\left(t_{d e l}\right)$ as $\rho_{i}=J_{0}\left(2 \pi f_{D i} t_{d e l}\right)$ [6], where $J_{0}$ is the zeroth-order Bessel function of the first kind.

Let $\mathbf{x}[t, n] \in \mathbb{C}^{n_{t} \times 1}$ be the $n_{t} \times 1$ transmitted vector at the $n$th time instant in the $t$ th time slot, while $y_{i}[t, n]$ denotes the $i$ th user's received signal at the same instant. The signal model is given by

$$
y_{i}[t, n]=\mathbf{h}_{i}(t) \mathbf{x}[t, n]+z_{i}[t, n]
$$




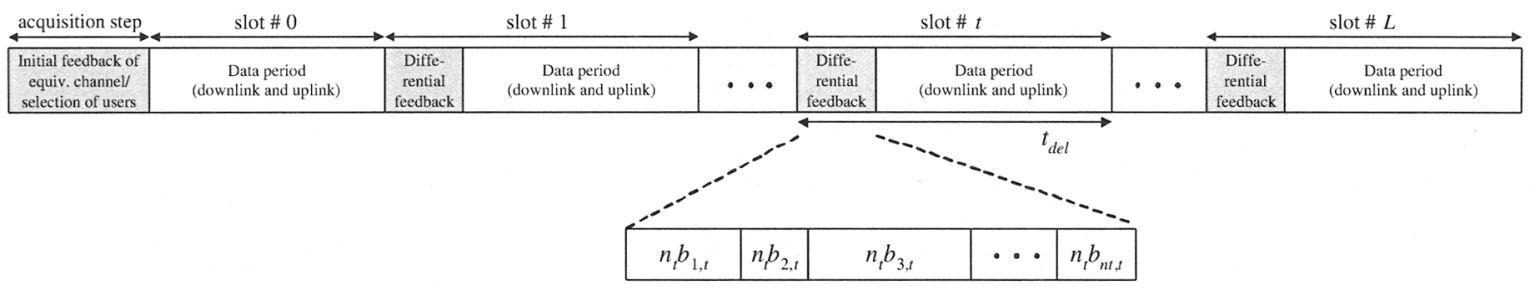

Fig. 1. Structure of the frame, including an initial feedback and acquisition stage and several slots containing differential feedbacks.

where $z_{i}[t, n]$ is an AWGN component with zero mean and variance $E\left[\left|z_{i}[t, n]\right|^{2}\right]=\sigma_{n}^{2}$. From now on and for simplicity in the notation, the time index will be dropped when possible.

\section{MULTIBEAM OPPORTUNISTIC BEAMFORMING}

One of the main transmission techniques in multiuser scenarios is MOB [1], where $n_{t}$ random orthonormal spatial beams $\left(\left\{\mathbf{b}_{i}\right\}_{i=1}^{n_{t}}\right)$ are generated at the BS to simultaneously serve more than one user. Within the acquisition stage (usually performed at the beginning of each frame, as seen in Fig. 1), each one of the $N$ users calculates the equivalent channel gain seen from each beam and feeds back all of them to the BS. Based on this information, the scheduler at the BS chooses the $n_{t}$ users to be served simultaneously (usually, assigning to each beam the user with the highest equivalent channel gain from that beam and according to SNIR based criteria). In the following, we assume that the group of selected users remains unchanged for the whole frame and, as far as notation is concerned, the selected users are numbered from 1 to $n_{t}$ according to the index of the assigned beam. Note that the scheduling strategy is not in the focus of this paper and, therefore, we assume that the best users are selected and given [1].

The transmitted signal is constructed using the symbols intended for the $n_{t}$ selected users and where these symbols are assumed to have unitary energy $\left(E\left[\left|s_{i}\right|^{2}\right]=1, \mathbf{s}=\left[s_{1}, \ldots, s_{n_{t}}\right]^{T}\right)$ :

$$
\mathbf{x}=\sum_{i=1}^{n_{t}} \mathbf{x}_{i}=\sum_{i=1}^{n_{t}} \mathbf{b}_{i} p_{i}^{1 / 2} s_{i}=\operatorname{Bdiag}(\mathbf{p})^{1 / 2} \mathbf{s},
$$

with $\mathbf{b}_{i}$ as the unit-norm beam assigned to the $i$ th selected user, $p_{i}$ as the assigned power to that beam, and $\mathbf{p}=\left[p_{1}, \ldots, p_{n_{t}}\right]^{T}$. The unitary matrix $\mathbf{B}=\left[\mathbf{b}_{1}, \ldots, \mathbf{b}_{n_{t}}\right]\left(\mathbf{B}^{H} \mathbf{B}=\mathbf{I}\right)$ is randomly generated, while $\operatorname{diag}(\mathbf{p})^{1 / 2}$ is a diagonal matrix with $p_{i}^{1 / 2}$ as its diagonal entries. Notice that this formulation is an upgraded version of [1], as a power loading $\mathbf{p}$ over the transmitted beams is incorporated.

The SNIR formulation for the $i$ th selected user, with $n_{t}$ transmitting beams, is given by

$$
\operatorname{SNIR}_{i}(\mathbf{p})=\frac{p_{i}\left|\mathbf{h}_{i} \mathbf{b}_{i}\right|^{2}}{\sigma_{n}^{2}+\sum_{j \neq i}^{n_{t}} p_{j}\left|\mathbf{h}_{i} \mathbf{b}_{j}\right|^{2}}=\frac{p_{i} c_{i, i}}{\sigma_{n}^{2}+\sum_{j \neq i}^{n_{t}} p_{j} c_{i, j}}
$$

where $\left|\mathbf{h}_{i} \mathbf{b}_{j}\right|^{2}=c_{i, j}$ denotes the equivalent channel seen by the $i$ th receiver with respect to the $j$ th beam $\mathbf{b}_{j}$. Note that the BS scheduler receives the equivalent channel moduli from each user, so that the BS can calculate the values of SNIR for all the users.

The presented feedback load is the minimum one to guarantee QoS for the users [2]. Even this scheme works with partial CSIT, the signalling load that it involves is still large for its consideration within realistic systems. In order to decrease this load, an alternative scheduling process is established in this paper, so that the same $n_{t}$ selected users are serviced over the $L+1$ time slots in the frame
(Fig. 1). Therefore, in a first instant, all users feed back their channel metrics $\left\{c_{i, j}\right\}$ and the best user for each transmit beam is selected; after that and for $L$ slots, only the selected users feed back their updated channel information $\left\{c_{i, j}\right\}$ to the BS [7] [8]. In order to further improve the performance, a differential feedback policy is implemented exploiting in this way the channel correlation between consecutive slots.

\subsection{QoS in MOB}

The requirement for a certain QoS is presented in terms of a minimum SNIR value per user while taking into account the cross interference terms that appear in MOB. The problem can then be formulated through a minimization of the total transmit power $\left(P_{t}\right)$ over all possible power loading vectors $(\mathbf{p})$ subject to minimum SNIR requirements $\left(\operatorname{snir}_{i}^{t h}\right)$ :

$$
\begin{array}{cl}
\underset{\mathbf{p}}{\operatorname{minimize}} & P_{t}(\mathbf{p}) \\
\text { subject to } & \operatorname{SNIR}_{i}(\mathbf{p}) \geq \operatorname{snir}_{i}^{t h}, \quad \forall i .
\end{array}
$$

Note that the total transmit power for MOB is expressed as $P_{t}(\mathbf{p})=$ $\operatorname{Tr}\left(\mathbf{B d i a g}(\mathbf{p}) \mathbf{B}^{H}\right)=\mathbf{1}^{T} \mathbf{p}=\sum_{i=1}^{n_{t}} p_{i}$ because of the unitary transmitter processing matrix $\mathbf{B}$, so that together with the consideration of the SNIR expression in (4), makes the optimization problem in (5) to particularize for MOB as

$$
\begin{array}{ll}
\underset{\mathbf{p}}{\operatorname{minimize}} & \mathbf{1}^{T} \mathbf{p} \\
\text { subject to } & p_{i} c_{i, i}-\operatorname{snir}_{i}^{t h}\left[\sigma_{n}^{2}+\sum_{j \neq i}^{n_{t}} p_{j} c_{i, j}\right] \geq 0, \forall i,
\end{array}
$$

that admits a closed form expression resulting from the fact that at the optimum point, the inequalities become equalities [9]:

$$
\mathbf{p}=\mathbf{K}^{-1} \mathbf{n}, \quad P_{t}(\mathbf{p})=\mathbf{1}^{T} \mathbf{p}=\mathbf{1}^{T} \mathbf{K}^{-1} \mathbf{n},
$$

where $\mathbf{K} \in \mathbb{R}^{n_{t} \times n_{t}}$ and $\mathbf{n} \in \mathbb{R}^{n_{t} \times 1}$ are given by

$$
\begin{aligned}
{[\mathbf{K}]_{i, j} } & = \begin{cases}c_{i, i}, & \text { if } i=j, \\
-c_{i, j} \operatorname{snir}_{i}^{t h}, & \text { if } i \neq j\end{cases} \\
\mathbf{n} & =\left[\sigma_{n}^{2} \operatorname{snir}_{1}^{t h}, \ldots, \sigma_{n}^{2} \operatorname{snir}_{n_{t}}^{t h}\right]^{T} .
\end{aligned}
$$

The obtained solution provides an easy approach to test for the feasibility of the problem, as a negative value of any entry of $p$ indicates the unfeasibility of the corresponding requirement. The related beams can then be switched off to decrease the interference levels.

\subsection{A Robust Power Allocation Scheme}

In practical wireless scenarios the channel information available at the transmitter is not perfect, mainly due to the quantized and outdated feedback. The design of a MOB scheme that not only requires 
partial CSIT, but also being robust to uncertainty in the partial information, is a challenging aspect. Motivated by this, the paper presents a power allocation which is robust against these imperfections.

Notice that in the MOB scheme the generation of the $\mathbf{B}$ matrix is independent of the channel information, so that regardless of the CSIT quality, B cannot be robustly designed. The only resource that remains to deliver a robust transmission is the power loading over the generated beams [2]. A possible way to deal with the CSIT imperfections is through the application of a worst-case robust design of the power loading, to guarantee that the QoS requirements are always met for any possible error in the knowledge of the channel metrics $\left\{c_{i, j}\right\}$ while minimizing the required transmit power.

Back to eq. (4) and considering that the measurements are imperfect, the SNIR reformulates as

$$
\operatorname{SNIR}_{i}(\mathbf{p})=\frac{p_{i}\left(c_{i, i}+\delta_{i, i}\right)}{\sigma_{n}^{2}+\sum_{j \neq i}^{n_{t}} p_{j}\left(c_{i, j}+\delta_{i, j}\right)},
$$

with $\delta_{i, j}$ being the error in the equivalent channel modulus information $c_{i, j}$ available at the BS (note that, from now on, $c_{i, j}$ represents the available imperfect channel information instead of the actual value). The error is assumed to be unknown but upper-bounded by $\epsilon_{i}$ for each user and with respect to all beams, i.e., $\left(\left|\delta_{i, j}\right| \leq \epsilon_{i}\right)$. As the feedback quantization is independently applied to each equivalent channel gain, then in general $\delta_{i, j} \neq \delta_{i, j^{\prime}}$ for $j \neq j^{\prime}$. As will be explained later, the uncertainty error bound $\epsilon_{i}$ is directly related to the quantization applied by each user in the feedback, and this is allowed to change with time (the time index will be neglected for simplicity in the notation but explicitly incorporated when required).

The power allocation has to be performed for all cases of uncertainty, thus, a worst case calculation is needed to guarantee the QoS fulfillment. The worst case SNIR, i.e., the lower bound of the actual SNIR (10) for any possible error in the channel moduli information, is given by the following expression [2]:

$$
\operatorname{SNIR}_{i}^{w c}(\mathbf{p})=\frac{p_{i}\left(c_{i, i}-\epsilon_{i}\right)}{\sigma_{n}^{2}+\sum_{j \neq i}^{n_{t}} p_{j}\left(c_{i, j}+\epsilon_{i}\right)},
$$

where the lowest and highest possible channel gains have been chosen for the desired and the interference signals, respectively. Using this, the robust power allocation design problem is written as

$$
\begin{array}{cl}
\underset{\mathbf{p}}{\operatorname{minimize}} & \mathbf{1}^{T} \mathbf{p} \\
\text { subject to } & p_{i}\left(c_{i, i}-\epsilon_{i}\right) \\
& -\operatorname{snir}_{i}^{t h}\left[\sigma_{n}^{2}+\sum_{j \neq i}^{n_{t}} p_{j}\left(c_{i, j}+\epsilon_{i}\right)\right] \geq 0, \forall i .
\end{array}
$$

The total transmit power resulting from this problem is given by

$$
\begin{aligned}
\mathbf{p} & =\left(\mathbf{K}-\sum_{i=1}^{n_{t}} \epsilon_{i} \mathbf{A}_{i}\right)^{-1} \mathbf{n}, \\
P_{t}\left(\left\{\epsilon_{i}\right\}\right) & =\mathbf{1}^{T} \mathbf{p}=\mathbf{1}^{T}\left(\mathbf{K}-\sum_{i=1}^{n_{t}} \epsilon_{i} \mathbf{A}_{i}\right)^{-1} \mathbf{n},
\end{aligned}
$$

which is obtained in a similar way as the result (7) for the case of perfect partial CSIT. Note that the result depends on the maximum errors $\left\{\epsilon_{i}\right\}$ through matrix $\mathbf{A}_{i}$, which is defined as

$$
\left[\mathbf{A}_{i}\right]_{m, n}= \begin{cases}\operatorname{snir}_{i}^{t h}, & \text { if } m=i, n \neq i \\ 1, & \text { if } m=i, n=i \\ 0, & \text { if } m \neq i\end{cases}
$$

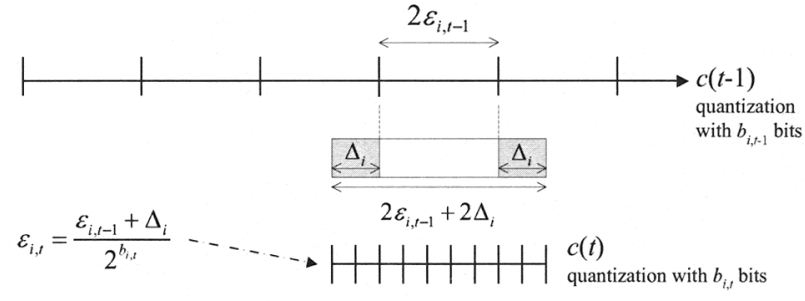

Fig. 2. Relation between the current and the previous differential quantization steps depending on the number of quantization bits and the enlargement due to the Doppler frequency.

where the feasibility of the problem is also checked through the signs of the entries of $\mathbf{p}$ in (13).

The closed form expression for the robust design allows to calculate the derivative of the transmit power with respect to $\epsilon_{i}$ as

$$
\frac{\partial P_{t}}{\partial \epsilon_{i}}\left(\left\{\epsilon_{i}\right\}\right)=\mathbf{1}^{T}\left(\mathbf{K}-\sum_{j=1}^{n_{t}} \epsilon_{j} \mathbf{A}_{j}\right)^{-1} \mathbf{A}_{i}\left(\mathbf{K}-\sum_{j=1}^{n_{t}} \epsilon_{j} \mathbf{A}_{j}\right)^{-1} \mathbf{n},
$$

which shows a key feature of this system, as it reflects the transmit power sensitivity to the uncertainty of each user measure independently (i.e., there may be users with a higher sensitivity to errors in the feedback quantization than other users). This sensitivity is later exploited in the design of a dynamic bit allocation problem in the feedback link in order to increase the system performance.

\section{DYNAMIC FEEDBACK BIT ALLOCATION}

As already mentioned, the main sources of uncertainty in the feedback comes from the channel variation and the fact that the equivalent channel moduli information $\left\{c_{i, j}\right\}_{i, j=1}^{n_{t}}$ available at the receivers has to be quantized before feeding it back to the BS. In this paper it is considered that the varying channel is temporally correlated between slots (see eq. (1)), which allows to employ a differential quantization [5] jointly with the fact that the set of selected users is kept fixed during the whole frame. This strategy allows to model the maximum error in the feedback for each user as

$$
\epsilon_{i, t}=\frac{\epsilon_{i, t-1}+\Delta_{i}}{2^{b_{i, t}}}
$$

where $\epsilon_{i, t}$ is the maximum error due to the feedback in the $t$ th block, $b_{i, t}$ is the number of bits allocated to the feedback of the $i$ th user in the $t$ th slot, and $\Delta_{i}$ accounts for the channel variation between consecutive slots and therefore, is related to both its temporal correlation $\rho_{i}$ and the speed of the $i$ th mobile user. Note that, if the channel was invariant $\left(\Delta_{i}=0\right)$, then the differential quantization would always imply a reduction of the uncertainty in the knowledge of the equivalent channel moduli. Although, as predicted by eq. (1), it is not possible to find a value for $\Delta_{i}$ such that the channel variation is bounded, there exists a value such that the variation lies in a predefined interval with a given high probability $P_{i n}$. As shown in the Appendix, the relationship between this probability $P_{i n}, \rho_{i}$, and $\Delta_{i}$ is given by

$$
\Delta_{i}=-\sigma_{h_{i}}^{2} \sqrt{1+\rho_{i}^{6}-\rho_{i}^{4}-\rho_{i}^{2}} \cdot \log \left(1-P_{i n}\right) .
$$

Fig. 2 shows an example of the relationship between the quantization steps in the current and the previous time slots and its dependance with the increase of uncertainty due to the channel variability. 
Assuming that the previous robust design of the power allocation is applied, the new transmit power at the $t$ th block after differential feedback can be linearly approximated as

$$
P_{t}\left(\left\{\epsilon_{i, t}\right\}\right) \simeq P_{t}\left(\left\{\epsilon_{i, t-1}\right\}\right)+\sum_{i=1}^{n_{t}} \frac{\partial P_{t}}{\partial \epsilon_{i}}\left(\left\{\epsilon_{i, t-1}\right\}\right)\left(\epsilon_{i, t}-\epsilon_{i, t-1}\right) .
$$

The feedback bits represent a very important and valuable resource in the system, since they are employed to guarantee QoS. This is the reason why a dynamic bit allocation among users is proposed in order to optimize the system efficiency and the tradeoff between the global feedback load and transmission power. This bit allocation should take into account the uncertainty sensitivity of each user (eq. (16)). In order to do that, the proposal consists in minimizing the total transmit power (19) while simultaneously fixing the total number of bits for feedback, i.e., $n_{t} \sum_{i=1}^{n_{t}} b_{i, t}=n_{b}$ (it is, for example, equivalent to assume a TDMA subslot for the feedback stage with a prefixed time duration). This dynamic bit allocation problem can be mathematically formulated as

$$
\begin{array}{ll}
\underset{\left\{b_{i, t}\right\}}{\operatorname{minimize}} & P_{t}\left(\left\{\epsilon_{i, t}\right\}\right) \\
\text { subject to } & n_{t} \sum_{i=1}^{n_{t}} b_{i, t}=n_{b} .
\end{array}
$$

The previous problem has integer variables represented by $\left\{b_{i, t}\right\}$ and, therefore, its optimum solution requires exhaustive search. Note, however, that a good approximation can be obtained by relaxing the problem and allowing the bit allocation to be continuous during the optimization and, afterwards, quantizing the result to the nearest integer [10]. If the previous optimization problem is solved by means of the Lagrange multipliers method, the following relationship is obtained:

$$
\frac{\partial P_{t}}{\partial \epsilon_{i}}\left(\left\{\epsilon_{i, t-1}\right\}\right) \frac{\epsilon_{i, t-1}+\Delta_{i}}{2^{b_{i, t}}}=\lambda,
$$

where the multiplier $\lambda$ is calculated so that the constraint in problem (20) is fulfilled. The previous expression provides an intuitive insight into the proposed dynamic bit allocation strategy for the differential quantization. As seen in (21), the number of awarded bits to the $i$ th user increases with $\frac{\partial P_{t}}{\partial \epsilon_{i}}$, so that if the system realizes that the required power is more sensitive to errors in the feedback of user $i$ than to user $j$, then, more bits would be allocated to the $i$ th user. On the other hand, the number of bits also increases with $\Delta_{i}$, which emphasizes that if a user has a fast varying channel due its mobility, then the number of bits allocated to such user would also increase.

Finally, note that in order to perform the optimum bit allocation, the proposed dynamic feedback bit allocation procedure is applied in a block-by-block basis, i.e., in each slot the partial derivatives of the total transmit power have to be re-calculated with the new values of the error bounds $\left\{\epsilon_{i, t}\right\}$

\section{SIMULATIONS RESULTS}

The performance of the proposed schemes is presented by Monte Carlo simulations, where the objective is two fold: to decrease both the feedback load and the transmit power, while ensuring the QoS restrictions in terms of minimum SNIR per served user. We consider a wireless scenario with $n_{t}=3$ transmit antennas in a cell with a variable number of active single-antenna receiving users, and a system bandwidth of $1 \mathrm{MHz}$. The transmitter runs the MOB technique where a total of 3 orthonormal beams are set up. A channel variance $\sigma_{h}^{2}=1$ is assumed for all users together with a temporal correlation

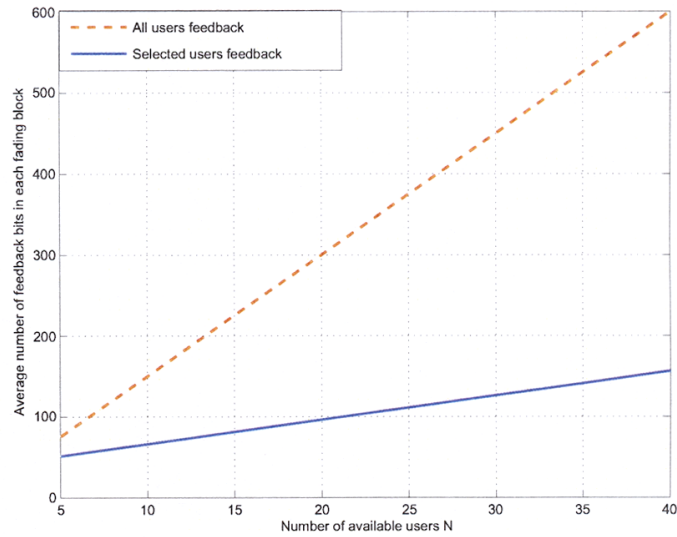

Fig. 3. Number of feedback bits in the different feedback policies.

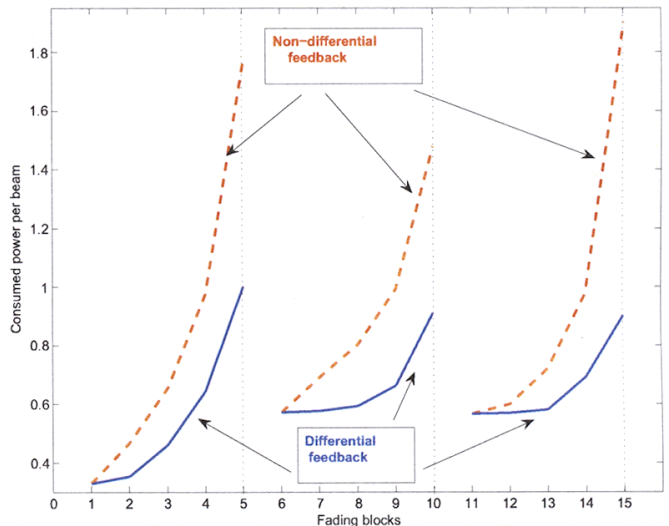

Fig. 4. Performance of the differential feedback policy in comparison to a non-differential one.

of $\rho_{i}=0.9$. A heterogeneous scenario is developed with users asking for different SNIR requirements so as to achieve minimum rate successful transmissions of $585 \mathrm{Kbps}, 1 \mathrm{Mbps}$, and $1.3 \mathrm{Mbps}$. The total number of feedback bits in the system is 45 bits, so that in the case of a fixed bits allocation, they are uniformly distributed among the users and beams. On the other hand for the dynamic policy, the bits are allowed to be non-uniformly distributed among the users, as can be seen in eqn. (20).

The feedback reduction of the proposed scheme is now presented, where in the first fading block, all users feed back their channel moduli information, but once the selection is accomplished, then only the selected users feed back their modified channel values. This feedback reduction is actually the same for all discussed schemes in this paper, and it is compared to the scenario where all users feed back their moduli values over each fading block. The results are presented in Fig. 3, where the large feedback load saving of the proposed schemes is shown, making the implementation of the MOB technique to be more viable in practical systems.

As the selected users are the same over all the $L$ fading blocks, then a differential feedback policy can be implemented, decreasing the uncertainty values. A lower uncertainty actually drives less power consumption at the transmitter side to guarantee the users' QoS requirements. Fig. 4 shows a temporal snapshot of 15 fading blocks with $L=5$, so that 3 different groups of selected users are presented in this figure. It plots the mean power consumption 


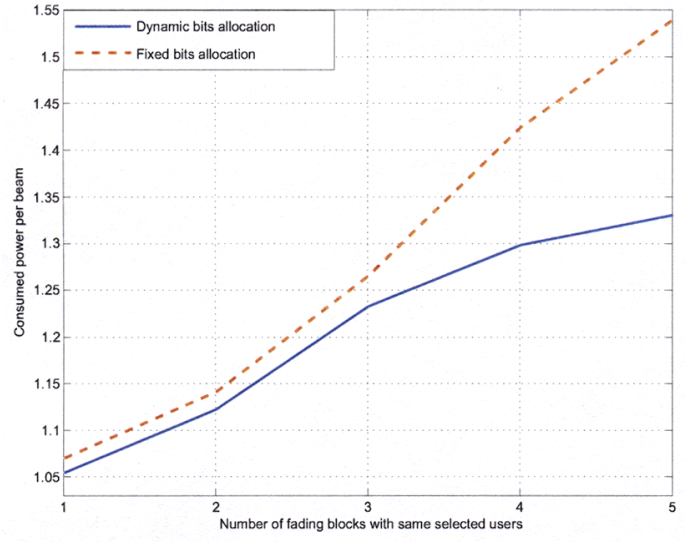

Fig. 5. The dynamic bits allocation strategy behaviour.

per beam to satisfy the QoS for the differential and non-differential quantization policies, showing a great power saving for the differential scheme as the transmitter faces with lower uncertainty measures.

Related to the dynamic bits allocation approach implemented on the differential feedback policy, Fig. 5 displays the power saving of this technique in comparison to a fixed or uniform bits allocation strategy, where both approaches exploit a differential quantization procedure. The results correspond to the average over a very large time sample and over $L=5$ fading blocks. The results show that with the dynamic policy, there exists a saturation effect in the sense that any changes in the instantaneous channel, following the model in eq. (1), are compensated by the dynamic bits allocation, while the fixed allocation still requires for more power to satisfy the QoS demands.

\section{CONCLUSIONS}

This paper has proposed a transmit power allocation technique for MOB which is robust against errors in the knowledge of the equivalent channel gains. These errors are generated due to the quantization applied to the CSI feedback in addition to the users' mobility and, therefore, delay in the acquisition of such CSI. The necessary transmit power has different sensitivities to the errors and the Doppler frequencies of the users. This motivates the derivation of a dynamic bits allocation among the users in the feedback stage in combination with a differential quantization approach. The simulations show that the proposed technique presents a good tradeoff between the total feedback load and the required transmission power when compared to other feedback policies, making the proposed scheme to be an attractive option for its implementation in practical systems.

\section{APPENDIX}

This Appendix provides a sketch of the proof for eq. (18), which measures the maximum shift of the equivalent channel gain due to the Doppler frequency with a given probability. This relationship depends on each user and the proof can be applied independently to each user in the system. Taking this into account, and for the sake of simplicity in the notation, the subindex corresponding to the user will be omitted.

The equivalent channel modulus at the $t$ th time slot is given by $c(t)=|\mathbf{h}(t) \mathbf{b}|^{2}$ (note that the subindexes have also been omitted in $c(t), \mathbf{h}(t)$, and $\mathbf{b}$ because the results and the proof do not depend on the considered beam and channel). Using the channel variation model in eq. (1), the following expression is obtained:

$$
\begin{aligned}
c(t) & =|\mathbf{h}(t) \mathbf{b}|^{2}=\left|\rho \mathbf{h}(t-1) \mathbf{b}+\sqrt{1-\rho^{2}} \mathbf{w}(t) \mathbf{b}\right|^{2} \\
& =\rho^{2}|\alpha|^{2}+\left(1-\rho^{2}\right)|\beta|^{2}+2 \rho \sqrt{1-\rho^{2}} \mathfrak{R e}\left(\alpha \beta^{*}\right), \\
\alpha & =\mathbf{h}(t-1) \mathbf{b}, \quad \beta=\mathbf{w}(t) \mathbf{b}, \quad c(t-1)=|\alpha|^{2},
\end{aligned}
$$

where $\alpha$ and $\beta$ are independent complex Gaussian random variables with zero-mean and variance $\sigma_{h}^{2}$ since $\|\mathbf{b}\|=1$. The objective is to calculate which is the maximum variation $\Delta$ such that

$$
\operatorname{Prob}(|c(t)-c(t-1)| \leq \Delta)=P_{i n} .
$$

Using the previous results, the following relations are obtained:

$$
c(t)-c(t-1)=\left[\begin{array}{ll}
\alpha^{*} & \beta^{*}
\end{array}\right]\left[\begin{array}{cc}
\rho^{2}-1 & \rho\left(1-\rho^{2}\right) \\
\rho\left(1-\rho^{2}\right) & 1-\rho^{2}
\end{array}\right]\left[\begin{array}{l}
\alpha \\
\beta
\end{array}\right]
$$

The matrix in the previous expression can be diagonalized using the unitary matrix $\mathbf{U}$ and with two eigenvalues equal to $\pm \mu$ ( $\mu=$ $\left.\sqrt{1+\rho^{6}-\rho^{4}-\rho^{2}}\right)$, obtained from the characteristic polinomial of the matrix. Using it, the following expression is obtained:

$$
c(t)-c(t-1)=\mu|\widetilde{\alpha}|^{2}-\mu|\widetilde{\beta}|^{2}, \quad\left[\begin{array}{c}
\widetilde{\alpha} \\
\widetilde{\beta}
\end{array}\right]=\mathbf{U}^{H}\left[\begin{array}{c}
\alpha \\
\beta
\end{array}\right],
$$

where again $\widetilde{\alpha}$ and $\widetilde{\beta}$ are independent complex Gaussian random variables with zero-mean and variance $\sigma_{h}^{2}$. Thus, $|\widetilde{\alpha}|^{2}$ and $|\widetilde{\beta}|^{2}$ are exponential and independent, allowing to conclude that $x=c(t)-$ $c(t-1)$ is distributed according to the following pdf:

$$
p_{X}(x)=\frac{1}{2} \frac{1}{\mu \sigma_{h}^{2}} \exp \left(-\frac{|x|}{\mu \sigma_{h}^{2}}\right), \quad-\infty<x<\infty,
$$

which, by a simple integration, involves that (24) is rewritten as

$$
\operatorname{Prob}(-\Delta \leq x \leq \Delta)=1-\exp \left(-\frac{\Delta}{\mu \sigma_{h}^{2}}\right)=P_{i n},
$$

and, therefore, result (18) is obtained.

\section{REFERENCES}

[1] M. Sharif and B. Hassibi, "On the Capacity of MIMO Broadcast Channel with Partial Side Information," IEEE Trans. on Inform. Theory, vol. 51, no. 2, February 2005.

[2] N. Zorba and A.I. Pérez-Neira, "Robust Multibeam Opportunistic Schemes Under Quality of Service Constraints," IEEE ICC, GlasgowUK, June 2007.

[3] N. Jindal, "MIMO Broadcast Channels with Finite Rate Feedback", IEEE Trans. on Inform. Theory, vol.52, November 2006.

[4] M. Kobayashi, G. Caire, and D. Gesbert, "Transmit Diversity vs. Opportunistic Beamforming in Data Packet Mobile Downlink Transmission", IEEE Trans. on Commun., vol.55, January 2007.

[5] J.L. Pan, R. Olesen, D. Grieco and C.P. Yen, "Efficient Feedback Design for MIMO SC-FDMA Systems", IEEE-VTC Spring, Dublin-Ireland, April 2007.

[6] R. Steele and L. Hanzo, Mobile Radio Communications, John Wiley and Sons, $2^{\text {nd }}$ Edition, New York, 1999.

[7] R. Zakhour and D. Gesbert, "A Two-Stage Approach to Feedback Design in MU-MIMO Channels with Limited Channel State Information", IEEE-PIMRC, Greece, September 2007.

[8] N. Zorba, A.I. Pérez-Neira and M.A. Lagunas, "A Reduced Complexity MIMO Broadcast Scheme: A Way between Opportunistic and Dirty Paper Implementation", Eusipco 2006, Florence-Italy, September 2006.

[9] M. Biguesh, S. Shahbazpanahi and A.B. Gershman, "Robust Downlink Power Control in Wireless Cellular Systems", EURASIP-JWCN, vol. 2004, no.2, December 2004

[10] D. Hughes-Hartogs, "Ensemble Modem Structure for Imperfect Transmission Media", US Patent 4-679-227, July 1987. 